\title{
On the recurrence time and outburst properties of the soft X-ray transient Aquila X-1*
}

\begin{abstract}
V. Šimon $\star \star$
Astronomical Institute, Academy of Sciences of the Czech Republic, 25165 Ondřejov, Czech Republic

Received 14 September 2001 / Accepted 15 October 2001

Abstract. The analysis of the recurrence time $T_{\mathrm{C}}$ of outbursts in Aql X-1, using the method of $\mathrm{O}-\mathrm{C}$ residuals, revealed that the character of the $\mathrm{O}-\mathrm{C}$ curves bears a striking similarity to that of dwarf novae. It means that variations of $T_{\mathrm{C}}$ are large, but generally not chaotic and long-term trends in the $\mathrm{O}-\mathrm{C}$ curves can be resolved. The prevailing value of $T_{\mathrm{C}}$ is 211 days but the evolution of $T_{\mathrm{C}}$ shows several large jumps. The mean $T_{\mathrm{C}}$ varies by more than 1:2 and the switches occur within just a single epoch. A comprehensive study of the morphology of the light curves from $A S M / R X T E$ revealed that although the respective outbursts in Aql X-1 differ in both their duration and the maximum intensity, their rising and decay branches display remarkable similarities. The rising branches are exponential. The slope of the decay is broken in some events, with the final phase having a steeper slope and being roughly linear. We argue that just the inner disk is irradiated during outburst and that viscous heating plays a big role. The evolution of the hardness ratios $H R 1$ and $H R 2$ displays common characteristics for the respective outbursts observed by $A S M / R X T E$. $H R 1$ at the peak intensity of the more intense outbursts tends to be harder. Similar behaviour is also apparent for $H R 2$. An asymmetric course (hysteresis) of $H R 2$ with respect to the moment of maximum intensity is apparent for some outbursts, the largest value of $H R 2$ occurring on the rising branch. The observed behaviour is discussed in terms of the thermal instability model and variations of the thermal and power-law spectral components.
\end{abstract}

Key words. stars: activity - binaries: close - binaries: general - stars: magnetic fields - stars: individual: Aql X-1

\section{Introduction}

Aql X-1/V 1333 Aql is a soft X-ray transient (SXT) whose discrete X-ray outbursts are accompanied by optical brightenings which can reach 14.8 mag $(V)$ (e.g. Charles et al. 1980). The previous analyses of the recurrence time $T_{\mathrm{C}}$ of outbursts in Aql X-1 were based on an automated period search. Priedhorsky \& Terrell (1984) carried out Fourier analysis and $\chi^{2}$ tests of the binned curves of the events within 1969-1975. They argued that there is an underlying cycle of 123 days which does not lead necessarily to every outburst. They also suggested a phase-smearing of the respective outbursts. Kitamoto et al. (1993) analyzed the timings of outbursts within 1969-1992 by calculating the standard deviation of observed phases from a trial period. They concluded that the original $T_{\mathrm{C}}$ changed from 125 days to 309 days.

Aql X-1 contains a neutron star (NS) because type I bursts were observed (Koyama et al. 1981). The orbital period is 18.71 hours (Chevalier \& Ilovaisky 1991; Welsh et al. 2000). The secondary star of spectral type K6-M0 is likely to fill its Roche lobe (Chevalier et al. 1999;

* This research has made use of the observations provided by the $A S M / R X T E$ team.

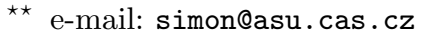

Welsh et al. 2000). The dimensions of Aql X-1 are therefore similar to the dwarf novae at the upper limit of the orbital periods of cataclysmic variables $(\mathrm{CVs})$, e.g. DX And $\left(P_{\text {orb }}=10.6 \mathrm{hr}\right)$ or GK Per $\left(P_{\text {orb }}=1.99\right.$ days $)$.

The outbursts of the SXTs are suggested to be due to the thermal instability in accretion disk, similar to that in dwarf novae (e.g. van Paradijs 1996). The conditions in the disk of the SXT are affected by the presence of the central source (the NS in Aql X-1), which acts as an irradiating source during outburst and modifies the disk structure.

A preliminary version of a part of this analysis was presented by Šimon (2001a).

\section{Sources of the data}

The recent outbursts of Aql X-1 within the years 1996-2001 were observed by the All Sky Monitor (ASM) onboard Rossi X-ray Timing Explorer (RXTE) (http://xte.mit.edu/). This monitor provides longterm observations of an unprecedented quality for this object. The data file contained the sum band intensities $I_{\text {sum }}(1.5-12 \mathrm{keV})$ and the hardness ratios $H R 1=I_{\mathrm{B}}(3-5 \mathrm{keV}) / I_{\mathrm{A}}(1.5-3 \mathrm{keV}), H R 2=$ $I_{\mathrm{C}}(5-12 \mathrm{keV}) / I_{\mathrm{B}}(3-5 \mathrm{keV})$. Only one-day means were used in order to increase the signal/noise ratio. 
This binning is sufficient because our analysis concentrates on the long-term activity of Aql X-1 and hence on features in the light curve on the time scale of days and longer. Modified Julian Date, used by RXTE, was transformed into the more often-used Julian Date. Hereafter it is used in the form of JD-2400000. The optical observations, available for the time interval covered by the $A S M / R X T E$ measurements, come mostly from IAU Circulars. They were obtained with a CCD, mostly in the $V$-band, and their expected uncertainty is smaller than 0.1 mag. They are displayed in Fig. 1a and enable a comparison of the X-ray and optical activity. The level brighter than 19 mag $(V)$ indicates an outburst.

The truncation limit for $H R 1$ and $H R 2$ was determined in the following way. The quoted uncertainties $\sigma_{\mathrm{q}}$ of both $H R 1$ and $H R 2$ were plotted versus $I_{\text {sum }}$ and their dependence on it was examined. It was found that $H R 1$ and $H R 2$ have quite small $\sigma_{\mathrm{q}}$ 's, almost independent of $I_{\text {sum }}$ for $\sigma_{\mathrm{q}} \leq 0.3 \mathrm{ct} / \mathrm{s}$. Only as one goes toward quite small $I_{\text {sum }}$ 's does the $\sigma_{\mathrm{q}}$ of most measurements rapidly increase. It was therefore decided to use only the $H R$ 's with $\sigma_{\mathrm{q}} \leq 0.3 \mathrm{ct} / \mathrm{s}$ for the following analysis. This criterion is applied to all the diagrams.

The timings of the previous outbursts of Aql X-1 till April 1992 were summarized by Kitamoto et al. (1993). The moments of the outburst maxima between the years 1992 and 1996 were determined from IAU Circulars and from Fig. 1 of Harmon et al. (1996). The inaccuracy of the timings derived from these mostly optical data is larger due to incomplete coverage and may amount to several tens of days. Nevertheless, this does not significantly influence our results. All these timings were then used for the analysis of the variations of $T_{\mathrm{C}}$.

\section{Data analysis}

\subsection{General description}

The sum band $A S M / R X T E$ X-ray light curve in Fig. 1b covers the years 1996-2001 and shows several bright, welldefined outbursts, rising from about $0 \mathrm{ct} / \mathrm{s}$ up to about $20-50 \mathrm{ct} / \mathrm{s}$. Typical duration of these events is $30-60$ days. The coverage is almost continuous, in most cases multiple observations were secured within a day. However, there is an interval of missing data shortly after the start of observation. There is strong evidence that an outburst occurred here because its final decay is clearly present. The individual bright outbursts, that is, those which reached $I_{\text {sum }}>15 \mathrm{ct} / \mathrm{s}$ at maximum, are shown in detail in Figs. 2 and 3. The $A S M / R X T E$ observations lead to a breakthrough in our knowledge of the activity of Aql X-1 because they enable us to study the structure of the bright outbursts. In addition, they also reveal three faint, but prolonged events, whose X-ray light curves have relatively flat profiles. Their X-ray intensity increased typically to about $5 \mathrm{ct} / \mathrm{s}$ above the quiescent level for several tens of days. Hereafter, they will be referred to as the faint outbursts (FOs) (Fig. 4). It is remarkable that fainter and brighter outbursts tend to alternate in Aql X-1.

The moment of the outburst maximum and the peak intensity, $I_{\max }$, were determined by fitting a polynomial of the 2 nd-4th degree to the upper part of $A S M$ outburst light curve. The typical error is 1-2 days. Even in the case of an incompletely covered curve this moment can be constrained to within about 20 days, that is much less than $T_{\mathrm{C}}$.

Notice in Fig. 1 that HR1 of the bright outbursts rises toward $I_{\max }$ and then decreases as the outburst fades. On the contrary, this behaviour is often not so apparent for $H R 2$.

The method used for the following analysis of the $\mathrm{X}$-ray outbursts in Aql X-1 is similar to the methods and procedures applied to some dwarf novae by Šimon (2000a,b,c).

\subsection{The outburst recurrence time and its variations}

The character of activity of Aql X-1, i.e. the discrete outbursts, lasting for several dozen days and separated by a much longer interval of quiescence, bears a large similarity to the behaviour of another transient - dwarf novae (DN), especially of U Gem-type. So it appears promising to apply the method of the $\mathrm{O}-\mathrm{C}$ residuals, successfully tested on DN, because the respective outbursts can easily be resolved in the light curve. The method of the $\mathrm{O}-\mathrm{C}$ residuals from some reference period (e.g. Vogt 1980) enables us to determine $T_{\mathrm{C}}$ in $\mathrm{DN}$ and to analyze its variations. This powerful method also removes the drawbacks of the widely used approach that is based on the measurements of separation of the individual outbursts. The method of the $\mathrm{O}-\mathrm{C}$ residuals is not sensitive to the exact length of the reference period and the $\mathrm{O}-\mathrm{C}$ diagram can be constructed even if there are gaps in the data and hence some missed the outbursts. A more detailed discussion of this method was given by Šimon (2000a). It is known from the previous analyses (Vogt 1980; Šimon 2000a) that $T_{\mathrm{C}}$ in a given dwarf nova can vary by a large amount. The standard period searches therefore often reveal nothing, especially when the duration of outburst is much shorter than $T_{\mathrm{C}}$. Nevertheless, the $\mathrm{O}-\mathrm{C}$ curves still show that in many cases the changes of $T_{\mathrm{C}}$ are not chaotic and the well-defined trends can be unambiguously resolved in the $\mathrm{O}-\mathrm{C}$ diagrams. Quite often, segments within which the mean $T_{\mathrm{C}}$ stays constant are separated by episodes of rapid change.

At the first step, before the method of the O-C residuals can be applied, a detailed insight into the character of the light curve and the range of possible $T_{\mathrm{C}}$ 's have to be obtained. The very dense coverage by the $A S M / R X T E$ observations and their high sensitivity are important for the identification of the FOs which, if omitted, would introduce a bias towards longer $T_{\mathrm{C}}$ 's. The separations of the consecutive events, marked in Fig. 1b, show that $T_{\mathrm{C}} \approx 200$ days is dominant prior to JD 2451400 . 

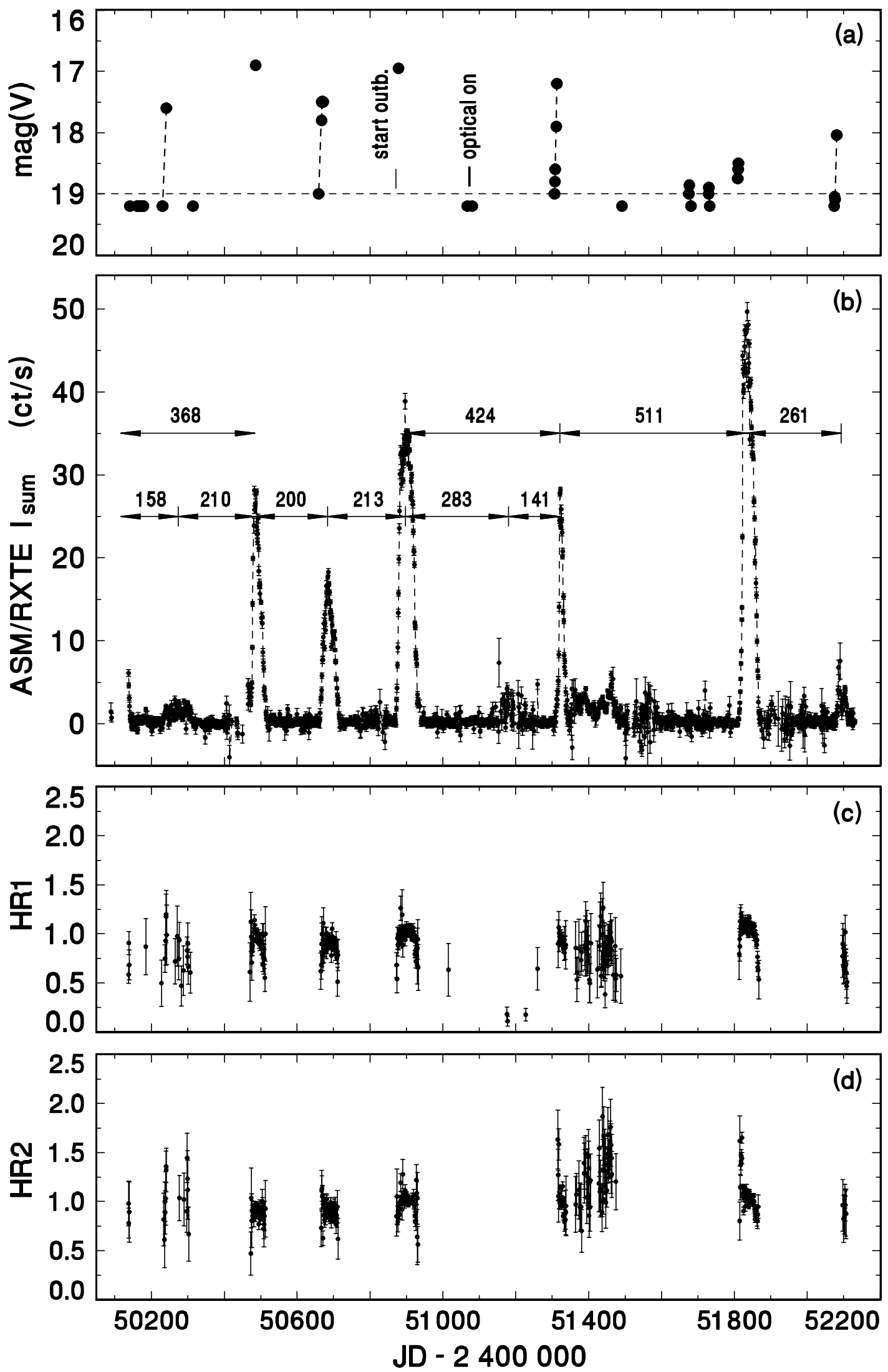

Fig. 1. The outburst history of Aql X-1 over the years 1996-2001 according to the $A S M / R X T E$ and optical observations. a) Optical observations. b) Sum band (2-12 keV) light curve. The points represent one-day means and are connected by the lines for the densely covered intervals. The arrows mark the separation between the consecutive events, discussed in the text. c) Hardness ratio $H R 1$. d) Hardness ratio HR2. The uncertainties quoted in the original file of the $R X T E$ measurements are marked. See Sect. 3.1 for details. 

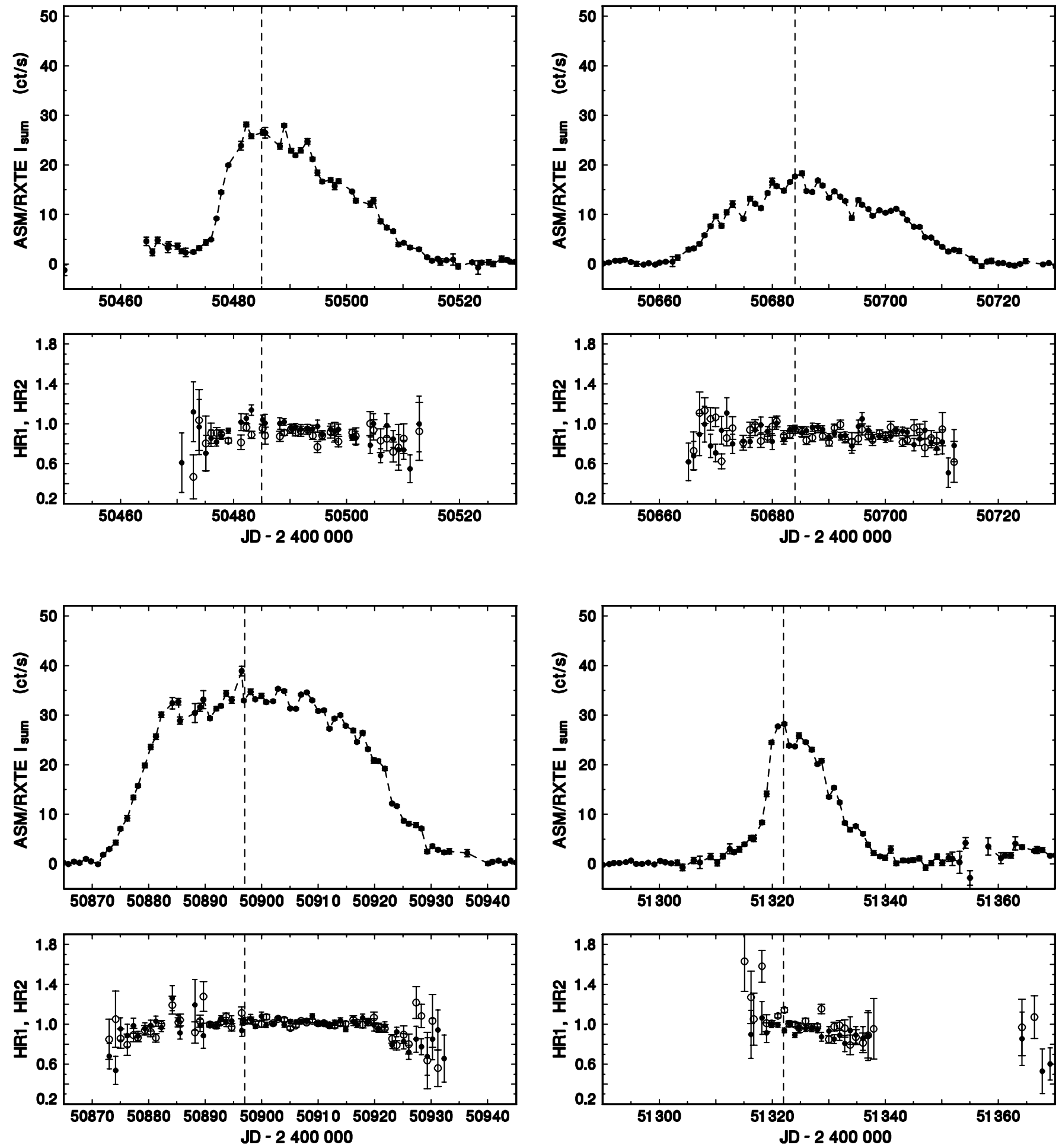

Fig. 2. The profiles of the bright outbursts in Aql X-1, based on the $A S M / R X T E$ observations. Sum band (2-12 keV) light curve, HR1 and HR2 are shown. The points represent one-day means and are connected by the lines for the densely covered intervals. The vertical line denotes the moment of $I_{\max }$. The scales of the axes are identical for all outbursts. See Sect.3.1 for details.

Fainter and brighter outbursts tend to alternate. Notice that for example the event between those at JD 2450484 and JD 2450897 is clearly fainter; a similar scheme can be applied also to the FOs. For the sake of completeness, intervals without taking the FOs into account are also marked. It is important that two FOs were reported to be accompanied by the optical brightenings with an amplitude comparable to the other events (Fig. 1a). The FOs were therefore included in the analysis of $T_{\mathrm{C}}$. 

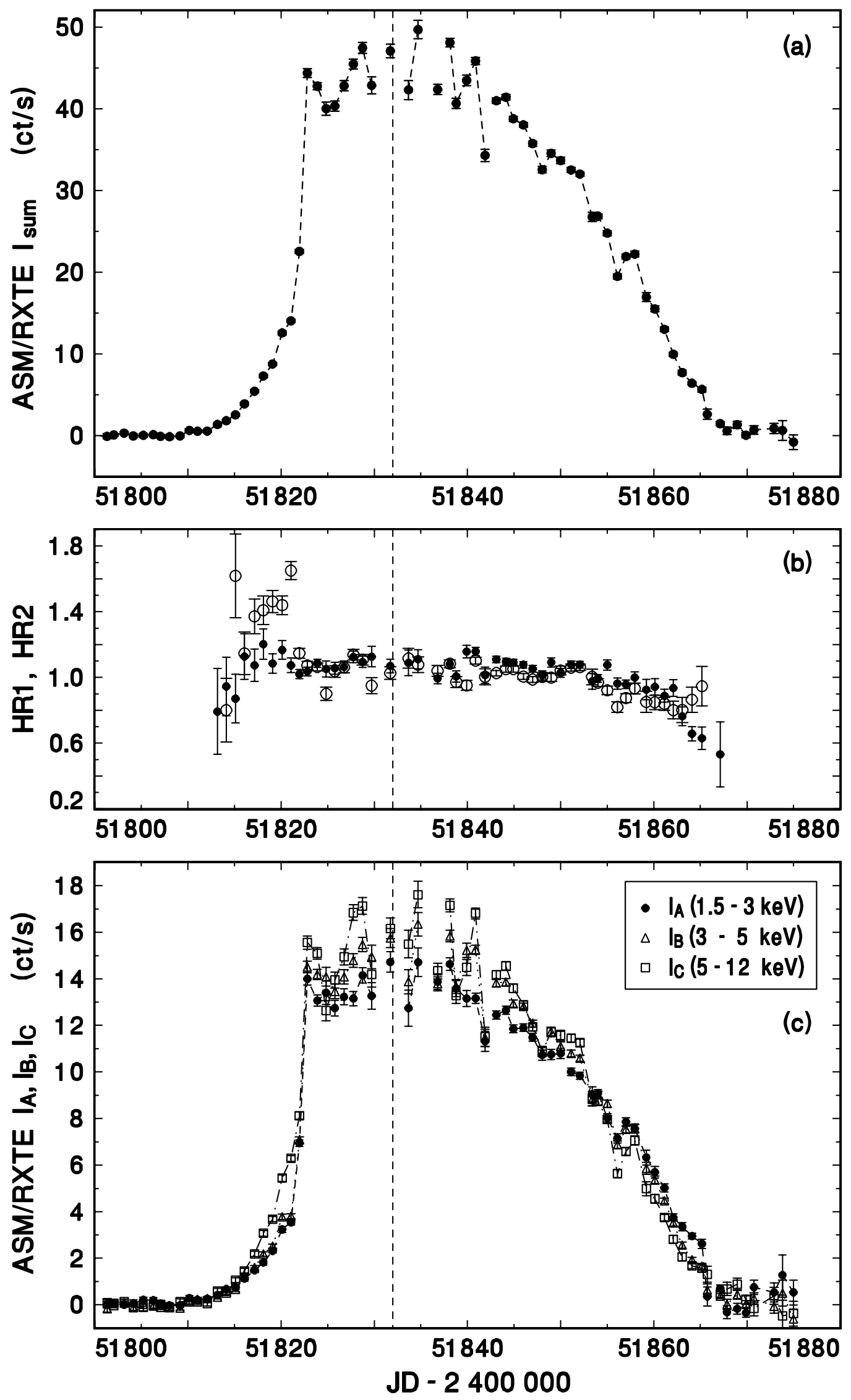

Fig. 3. The profile of the bright outburst in Aql X-1, having its maximum in JD 2451832. The arrangement of Figs. a) and b) is the same as that of Figs. 2. Notice that HR2 attains its highest value during the rise to $I_{\max }$ of outburst. The evolution of the outburst in the respective $A S M$ passbands is shown in Fig. c). See Sects. 3.3 and 3.4 for details. 

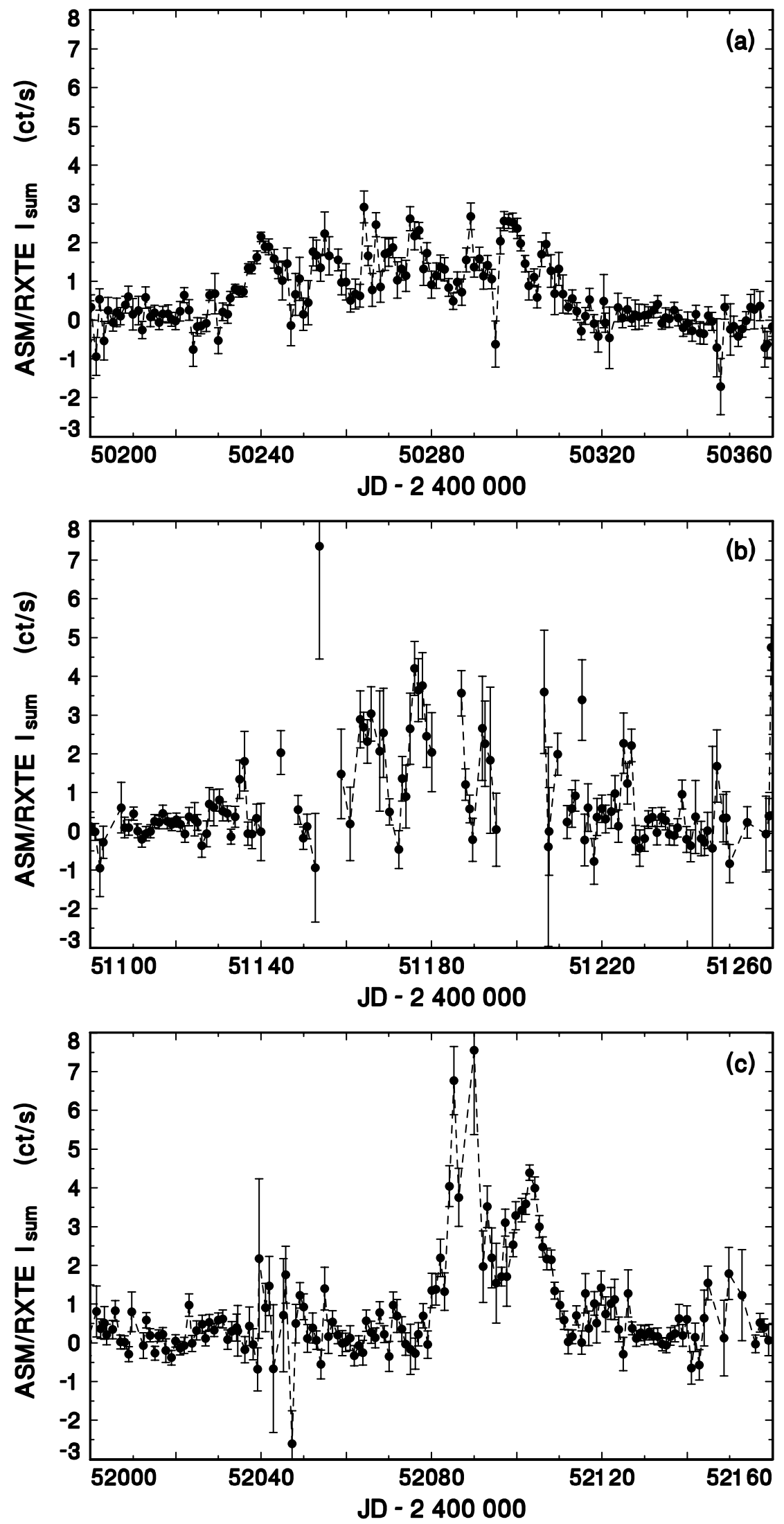

Fig. 4. The profiles of three faint outbursts (FOs) in Aql X-1, based on the $A S M / R X T E$ observations. The points represent one-day means and were connected by the lines in the densely covered parts of the curves. The scale of the abscissa is identical for all three plots. See Sect. 3.3 for details. 


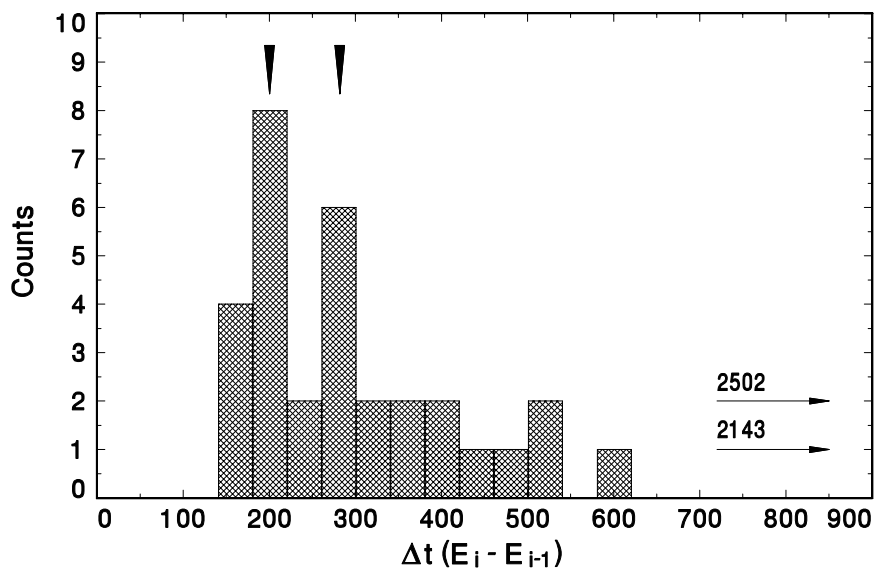

Fig. 5. The statistical distribution of the separation of the $o b$ served consecutive outbursts of Aql X-1 over the years 19692001. Notice the prominent peak at approx. 200 days and a secondary peak at approx. 300 days.

In order to obtain some starting value of $T_{\mathrm{C}}$, the separation of all observed consecutive outbursts of Aql X-1 over the years 1969-2001 was determined. The statistical distribution of these intervals is shown in Fig. 5. This histogram allows a good estimate of $T_{\mathrm{C}}$ to be obtained. For example, in the case that $T_{\mathrm{C}}$ remains constant and only some outbursts were missed for some intervals due to the absence of data then the histogram will display several peaks, corresponding to the value of the true $T_{\mathrm{C}}$ and its multiples. Figure 5 shows a flatter distribution than this ideal case but still a prominent peak at approx. 200 days and a secondary peak near 300 days can easily be resolved. These two values appear to be the promising lengths of the true $T_{\mathrm{C}}$. Notice that there is a clear cut-off at about 150 days and no hint of any short $T_{\mathrm{C}}$, like e.g. 125 days reported before. The tail toward longer $T_{\mathrm{C}}$ 's can be at least partly caused by the gaps in the data and hence by missed outbursts.

A careful examination of the $\mathrm{O}-\mathrm{C}$ diagrams, generated for various $T_{\mathrm{C}}$ 's and the basic maxima $T_{0}$ to obtain the mean slope of the $\mathrm{O}-\mathrm{C}$ values as small as possible, revealed that there are several intervals with quite different recurrence times. The variations of $T_{\mathrm{C}}$ in Aql X-1 were even found to be so large that they cannot be displayed in a single $\mathrm{O}-\mathrm{C}$ diagram. The reason is that the values of $T_{\mathrm{C}}$ 's, used in the ephemerides (Eqs. (1)-(4)), have to be so divergent that several outbursts fall into a single calculated epoch $E$. The $\mathrm{O}-\mathrm{C}$ diagram therefore becomes distorted in some segments. The $\mathrm{O}-\mathrm{C}$ curves were therefore generated for the data just inside the time intervals within which the distortions of the course of the $\mathrm{O}-\mathrm{C}$ values could be avoided. The Julian Dates, labeled for some points in Fig. 6, allow an easy orientation in the plots.

$$
\begin{aligned}
& T_{\max }=2441442+211 \mathrm{E} \\
& T_{\max }=2442572+380 \mathrm{E} \\
& T_{\max }=2447792+311 \mathrm{E}
\end{aligned}
$$

$T_{\max }=2450484+211 E$.

The timings and $\mathrm{O}-\mathrm{C}$ values of the individual outbursts are summarized in Table 1. The outbursts within JD 2440420-JD 2441687 can be folded with $T_{\mathrm{C}}=211$ days (Eq. (1)). The course of the $\mathrm{O}-\mathrm{C}$ values can be considered as linear (i.e. $T_{\mathrm{C}}$ is constant) with the standard deviation of 21 days (Fig. 6a). Notice that there is no missing outburst at the positions calculated according to Eq. (1); that speaks in favour of a correctly determined $T_{\mathrm{C}}$. However, a large abrupt change of $T_{\mathrm{C}}$ occurred after JD 2441687 and the recurrence time increased considerably. A careful examination of the $\mathrm{O}-\mathrm{C}$ diagram, calculated for several trial ephemerides, revealed that the interval within JD 2441687-JD 2443156 can be plausibly interpreted as a strong parabolic trend (Fig. 6b). There is just a slight difference between the fit by the 2 nd and the 3rd order polynomial, the standard deviations of the residuals being 23 and 21 days, respectively. A decrease of $T_{\mathrm{C}}$ can be easily resolved in Fig. $6 \mathrm{c}$ and can be interpreted as either abrupt or gradual. Also the interval in Fig. 6d, starting in JD 2449950, which displays a prevailing trend of a large increase of $T_{\mathrm{C}}$, can be interpreted in a similar way. The outburst BO5 had a complicated X-ray light curve with two rebrightenings. The circle in Fig. 6 d refers to the main peak but for the sake of completeness the $\mathrm{O}-\mathrm{C}$ value of the middle of the whole event is marked by a square.

In summary, it can be seen that the method of residuals enabled us to determine $T_{\mathrm{C}}$ and its large variations in Aql X-1 in spite of several missing outbursts which fall into intervals of poor coverage of the light curve.

The evolution of $T_{\mathrm{C}}$ in Aql X-1 could be determined from the course of the $\mathrm{O}-\mathrm{C}$ values in Fig. 6 . The result is shown in Fig. 7. Time is expressed in JD instead of epochs. This approach is inevitable because the lengths of $T_{\mathrm{C}}$ are often very discordant for the respective intervals. Time cannot be expressed in epochs in such a case. The horizontal straight lines represent the segments within which the mean $T_{\mathrm{C}}$ can be considered as constant. They were determined from the linear fits in Fig. 6. The tilted dashed lines, the slopes of which were derived from the quadratic fits, denote the intervals where the mean $T_{\mathrm{C}}$ can be approximated as steadily decreasing. The maxima of the two last outbursts, marked with the dots in Fig. 7, are affected by the large and well documented changes of $T_{\mathrm{C}}$.

\subsection{Morphology of the outburst light curves}

The $A S M / R X T E$ observations allow the features on the X-ray light curves of the bright outbursts in Aql X-1 to be resolved. A study of the morphology of these curves, mainly of their rising and decay branches, can best be made by matching the respective events to a template. The procedure used for an analysis of these transient events is similar to some extent to the method applied to the outbursts of the dwarf nova $\mathrm{CH}$ UMa by Simon (2000a). This approach is very efficient in a search for the common properties of the respective outbursts in a 

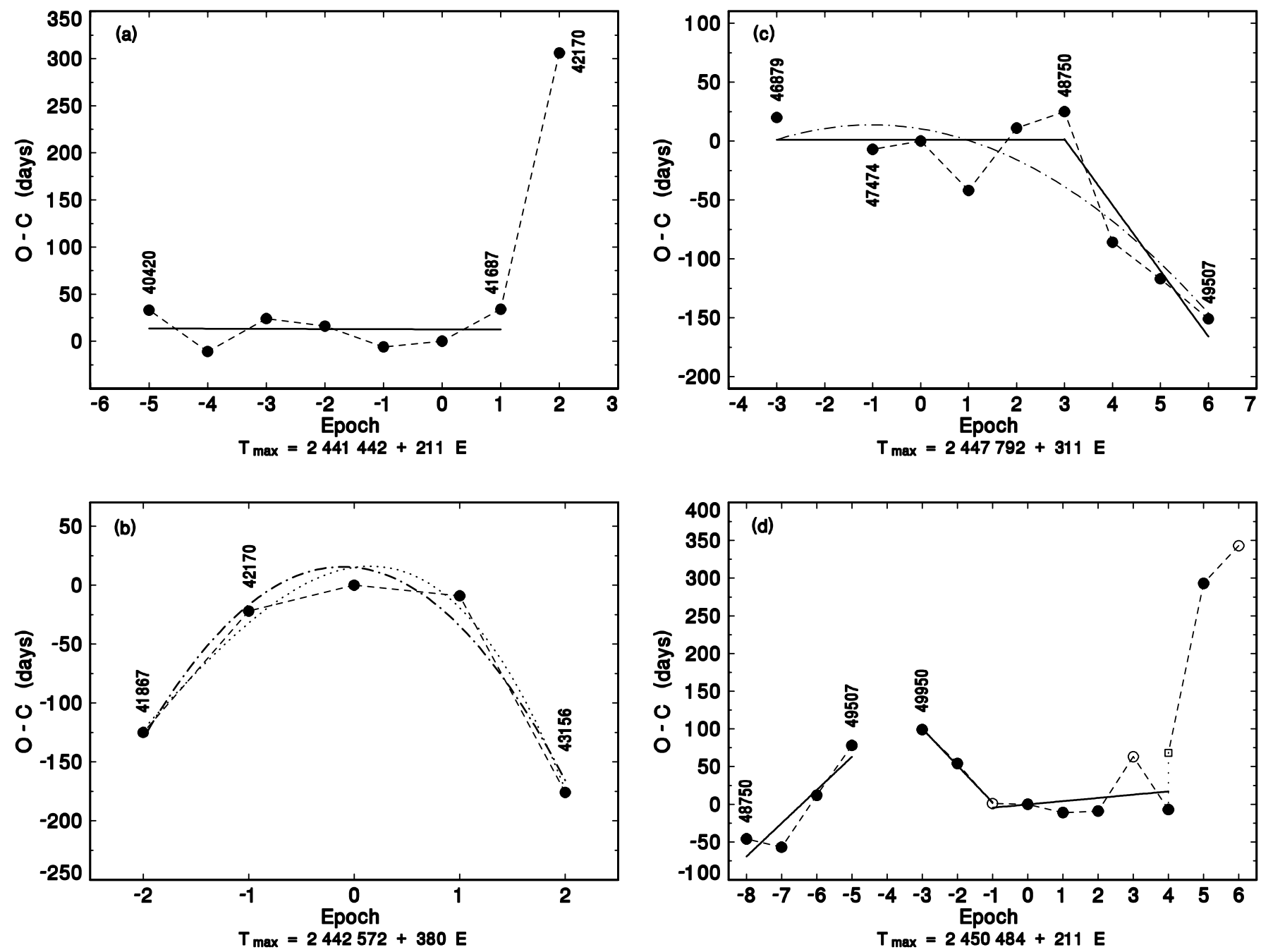

Fig. 6. $\mathrm{O}-\mathrm{C}$ diagram for the moments of the outburst maxima in Aql X-1. The variations of $T_{\mathrm{C}}$ are so large that they cannot be displayed in a single diagram. The $\mathrm{O}-\mathrm{C}$ values in the respective plots were calculated according to Eqs. (1)-(4). The outbursts that can be considered as consecutive are connected by the dashed lines. The thick straight lines denote the linear fits to the segments of the O-C curve. The dot-dashed and dotted curves represent the quadratic and cubic fits, respectively. The Julian Dates, labeled for some points, allow an easy orientation in the plots. See Sect. 3.2 for details.

given system. First, we analyzed the properties of the decay branches of the outbursts. The outburst having maximum in JD 2451832 (BO6 in Table 1) (Fig. 3) was chosen as the template because it reached the highest $I_{\max }$ of all the events. The remaining outbursts from Fig. 2 were shifted along the time axis to match the decay branch of the template. An examination of the light curves revealed that the slope of the decay is broken in some events, with the final phase having a steeper slope. $I_{\text {sum }}=10 \mathrm{ct} / \mathrm{s}$ was therefore chosen as the reference level, in the vicinity of which the match of the final phase of the decays was attempted. The result is shown in Fig. 8a. The decay branches of the individual outbursts were then merged into a common file and smoothed by the code HEC13, written by Dr. P. Harmanec and based on the method of Vondrák $(1969,1977)$. This code can fit a smooth curve to the data no matter what their course is. The fit with $\epsilon=10^{-1}, \Delta T=5$ days was found to satisfy the course of the decay. The residuals of the respective events show just a small scatter (Fig. 8b). The standard deviation of the residuals of this fit is just $1.2 \mathrm{ct} / \mathrm{s}$. The smoothed decay light curve is plotted as the thick solid line in Fig. 8a. It can be seen that a large part of the decay is almost linear. For the sake of completeness, the light curves of the outbursts with $I_{\text {sum }}$ on the logarithmic scale are displayed in Fig. 8c. This diagram confirms that the deviation from the exponential is really substantial for $I_{\text {sum }}>10 \mathrm{ct} / \mathrm{s}$ but it may be a plausible approximation below this value.

An examination of the light curves in Figs. 2 and 3 revealed that especially the lower part of the rise is rounded and resembles the exponential curve in most cases. This profile is particularly prominent in the outburst BO6 (Fig. 3). The rising branches were analyzed in a similar way as the decays (Fig. 9). The respective outbursts were shifted along the time axis to match the template but the time of crossing $5 \mathrm{ct} / \mathrm{s}$ was chosen in this case. 
Table 1. Timings of the outbursts in Aql X-1. The bright and faint outbursts, detected by $A S M / R X T E$, are abbreviated as $\mathrm{BO}$ and FO, respectively, in the column labeled Type. $T_{\max }$ refers to the moment of $I_{\max }$ in JD-2400000. The epoch number and $\mathrm{O}-\mathrm{C}$ (days) are calculated according to Eqs. (1)(4); the number of the equation used is listed in the column labeled equation. The $\mathrm{O}-\mathrm{C}$ variations are so large that in some cases several equations were used for the same outburst to make orientation in Figs. 6a, b, c easy.

\begin{tabular}{|c|c|c|c|c|c|c|c|}
\hline Type & $T_{\max } \mathrm{JD}$ & Epoch & $\mathrm{O}-\mathrm{C}$ & Eq. & Epoch & $\mathrm{O}-\mathrm{C}$ & Eq. \\
\hline & 40420 & -5 & 33 & (1) & & & \\
\hline & 40587 & -4 & -11 & (1) & & & \\
\hline & 40833 & -3 & 24 & (1) & & & \\
\hline & 41036 & -2 & 16 & (1) & & & \\
\hline & 41225 & -1 & -6 & (1) & & & \\
\hline & 41442 & 0 & 0 & (1) & & & \\
\hline & 41687 & 1 & 34 & (1) & -2 & -125 & $(2)$ \\
\hline & 42170 & -1 & -22 & $(2)$ & 2 & 306 & $(1)$ \\
\hline & 42572 & 0 & 0 & (2) & & & \\
\hline & 42943 & 1 & -9 & (2) & & & \\
\hline & 43156 & 2 & -176 & (2) & & & \\
\hline & 46879 & -3 & 20 & (3) & & & \\
\hline & 47474 & -1 & -7 & (3) & & & \\
\hline & 47792 & 0 & 0 & (3) & & & \\
\hline & 48061 & 1 & -42 & (3) & & & \\
\hline & 48425 & 2 & 11 & (3) & & & \\
\hline & 48750 & 3 & 25 & (3) & -8 & -46 & $(4)$ \\
\hline & 48950 & 4 & -86 & (3) & -7 & -57 & $(4)$ \\
\hline & 49230 & 5 & -117 & (3) & -6 & 12 & $(4)$ \\
\hline & 49507 & 6 & -151 & (3) & -5 & 78 & (4) \\
\hline & 49950 & -3 & 99 & (4) & & & \\
\hline BO1: & 50116 & -2 & 54 & (4) & & & \\
\hline FO1 & 50274 & -1 & 1 & (4) & & & \\
\hline BO2 & 50484 & 0 & 0 & (4) & & & \\
\hline BO3 & 50684 & 1 & -11 & (4) & & & \\
\hline $\mathrm{BO} 4$ & 50897 & 2 & -9 & (4) & & & \\
\hline FO2 & 51180 & 3 & 63 & (4) & & & \\
\hline BO5 & 51321 & 4 & -7 & (4) & & & \\
\hline BO6 & 51832 & 6 & 82 & (4) & 5 & 293 & (4) \\
\hline FO3 & 52093 & 6 & 343 & (4) & & & \\
\hline
\end{tabular}

In summary, our approach has clearly shown the fact that although the respective outbursts in Aql X-1 differ in their duration and $I_{\max }$ their rising and decay branches display remarkable similarities.

\subsubsection{Comparison with the 1978 outburst}

Comparison of the X-ray light curve of the 1978 outburst, observed by Ariel 5 ASM in the $3-6 \mathrm{keV}$ passband (Charles et al. 1980; Chen et al. 1997), with the recent events was made. The outburst BO6 was used as the representative of the events, observed by $A S M / R X T E$, because it attained the highest $I_{\max }$. The intensities were transformed into Crab units in order to enable a meaningful comparison of the events observed with the different instruments. In order to determine meaningful light curve of the 1978 outburst, the data were fitted by the

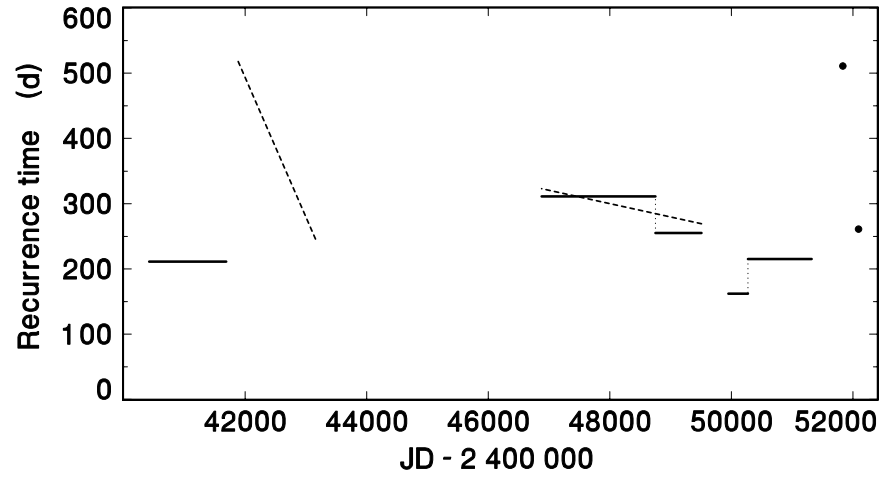

Fig. 7. The evolution of the mean $T_{\mathrm{C}}$ (in days) of the outbursts in Aql X-1, determined from Fig. 6. The horizontal straight lines represent the segments within which the mean $T_{\mathrm{C}}$ can be considered as constant. The intervals within which the mean $T_{\mathrm{C}}$ can be considered as steadily decreasing are marked by the tilted dashed lines and were determined from the quadratic fits in Fig. 6. See Sect. 3.2 for details.

above-mentioned code HEC13, with $\epsilon=10^{-1}$ and $\Delta T=$ 7 days. The fit from Fig. 8a was used for BO6. The same procedure as in Sect. 3.3 was made. The 1978 outburst was shifted along the time axis to match the decay branch of BO6. Although the noise of the 1978 outburst is large it can be seen that the decay branches of both events are not markedly different (Fig. 10).

\subsection{X-ray color behaviour}

All the available $H R 1$ 's and $H R 2$ 's of the outbursts of Aql $\mathrm{X}-1$ were plotted versus $I_{\text {sum }}$ in Fig. 11 . These data were divided into four subsets. The moment of $I_{\max }$, determined in Sect. 3.1, was chosen as the division point for the separation of the rising and decay branches of the bright outbursts. The rebrightening of $\mathrm{BO} 5$ and the FOs are also shown. There is a well-defined trend of hardening of both $H R 1$ and $H R 2$ with the intensity for $I_{\text {sum }}>10 \mathrm{ct} / \mathrm{s}$. The large empty circles in Fig. 11 represent the values, averaged around the peaks of the individual bright outbursts. Notice that the more intense outbursts tend to be harder at maximum (particularly their $H R 1$ ). On the other hand, an appreciable scatter of $H R 1$ and $H R 2$ which cannot be explained just by the observational noise is apparent for $I_{\text {sum }}<10 \mathrm{ct} / \mathrm{s}$. The scatter of $H R 1$ is roughly symmetrical with respect to the value of $H R 1$ just above $I_{\text {sum }}=10 \mathrm{ct} / \mathrm{s}$. On the other hand, the scatter of $H R 2$ extends significantly more to the harder values and is caused mainly by the rebrightening of $\mathrm{BO} 5$ and the rising branches of some bright outbursts.

The divergent behaviour of $H R 1$ and $H R 2$ during the rise to outburst and an asymmetry between the rising and decay branch were observed in several events. They were particularly prominent in the case of $\mathrm{BO} 6$, the brightest outburst observed with $A S M / R X T E$ (Fig. 12). Notice the pronounced asymmetry (hysteresis) of both $H R 1$ and $H R 2$ when the rise and decay are compared, mainly for $I_{\text {sum }}<20 \mathrm{ct} / \mathrm{s}$. HR2 especially achieves very high 

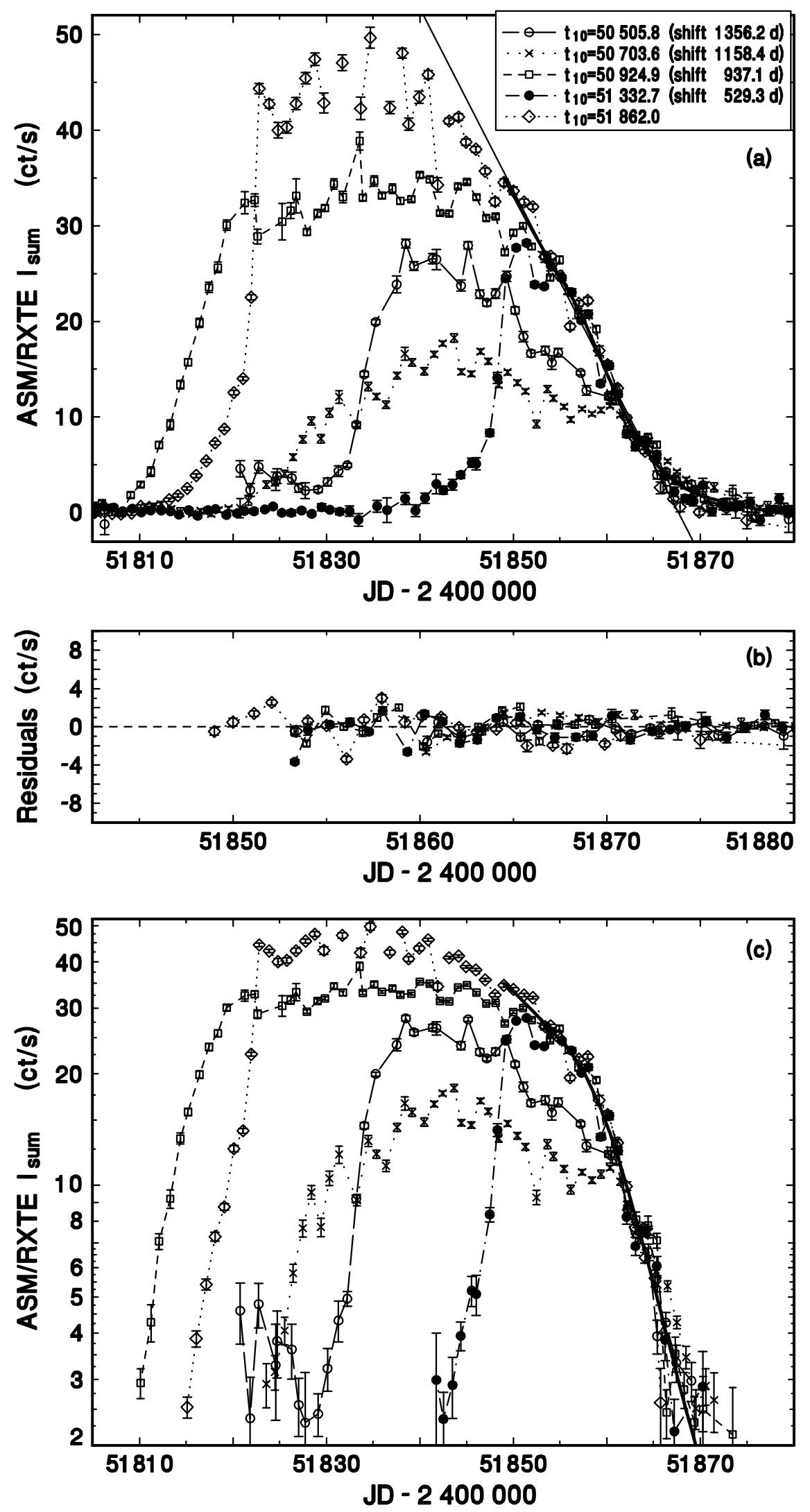

Fig. 8. a) comparison of the decay branches of the individual outbursts in Aql X-1, based on the $A S M / R X T E$ observations. The one-day means are shown. The individual outbursts were shifted along the time axis to match the decay branch of the template - the time of crossing $10 \mathrm{ct} / \mathrm{s}$ and the shifts with respect to the template are listed in the figure. The thick curve represents the smooth decay curve. The tilted line denotes the part of the decay branch which can be considered as linear. b) residuals of the fit to the decay. The scale of the abscissa is twice as large as in Fig. a). c) The same as in Fig. a) but for the logarithmic scale of the ordinate. See Sect. 3.3 for details. 

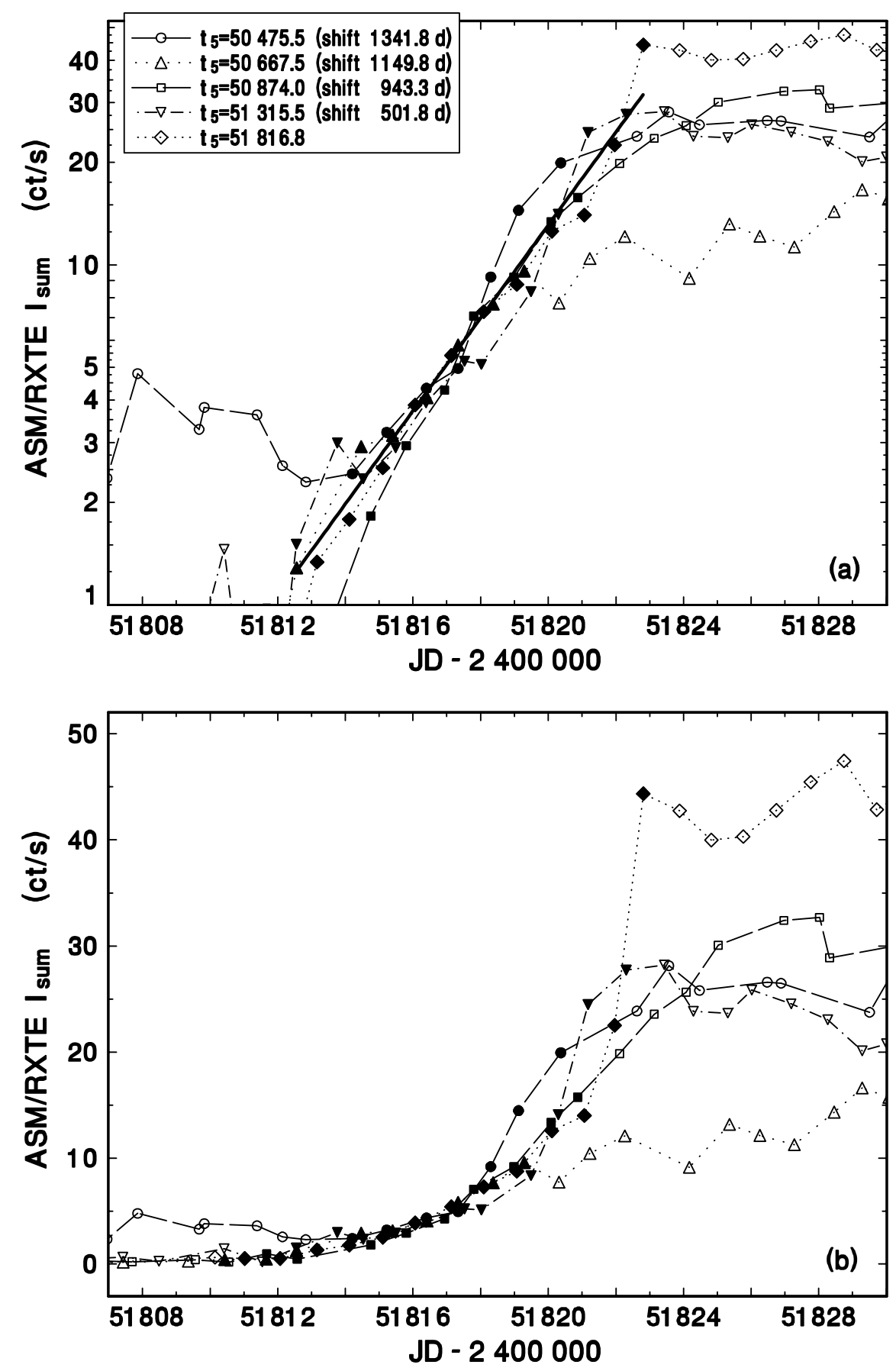

Fig. 9. The rising branches of the bright outbursts in Aql X-1, based on the $A S M / R X T E$ observations. The logarithmic and linear scale of the ordinate are used in Figs. a) and b), respectively. The individual outbursts were shifted along the time axis to match the rising branch of the template - the moment of crossing $5 \mathrm{ct} / \mathrm{s}$ and the shifts with respect to the template are listed. The tilted line in Fig. a) denotes the fit to the part of the decay branch which can be considered as exponential; the filled symbols mark the observations included in this fit. See Sect. 3.3 for details.

values at the onset of the outburst. Although the error bars are larger here than later at higher $I_{\text {sum }}$ the course is well defined and cannot be caused by the observational noise. The discrepancy between the rise and decay is more pronounced for HR2 than HR1. Further insight into what happened on the rising branch can be obtained from Fig. 3c where the light curves in the individual $A S M / R X T E$ passbands are shown. The intensity in the $\mathrm{C}$ channel $(5-12 \mathrm{keV})$ temporarily began to rise faster than in the other channels in the lower part of the rising branch.

An X-ray color-color diagram was constructed from the data, plotted in Fig. 13. In order to avoid overcrowding, the diagram is divided into four panels. It appears that the four groups differ from each other more by their $H R 2$ 's than $H R 1$ 's. 


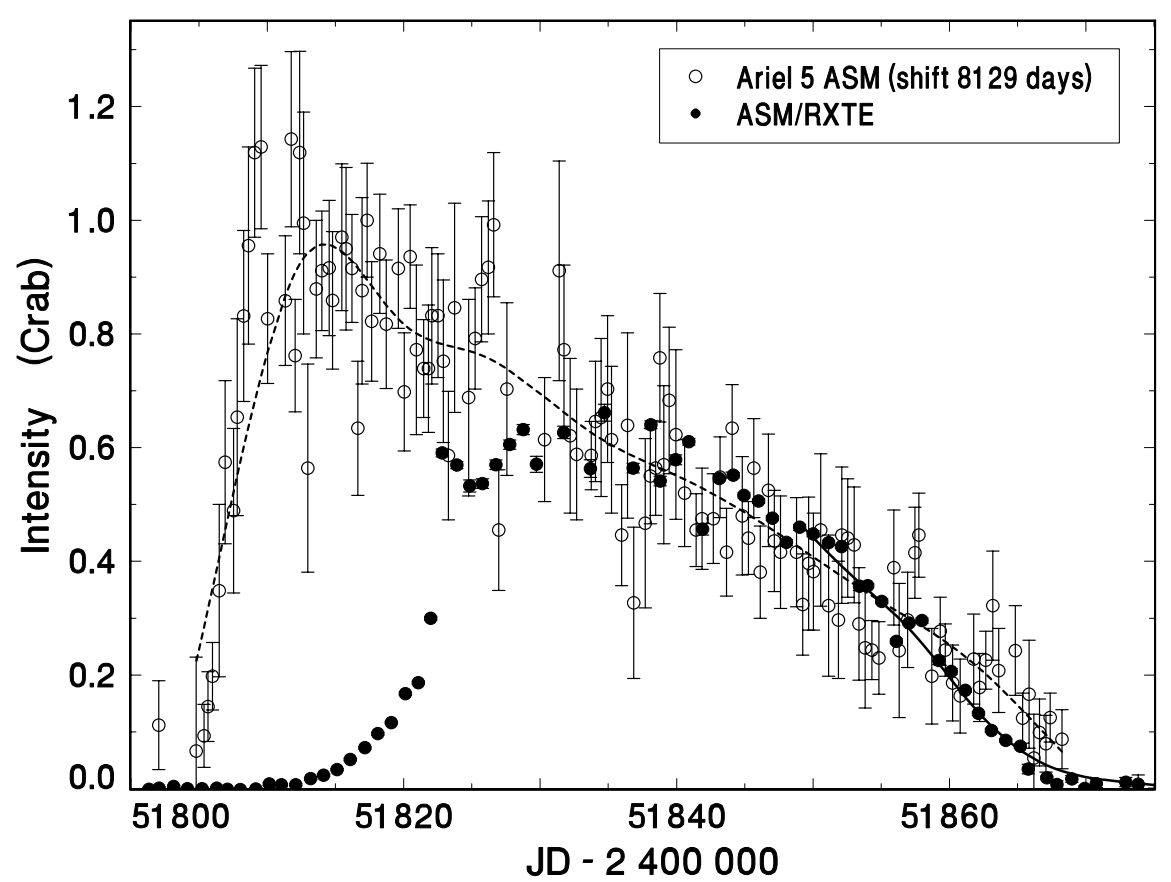

Fig. 10. Comparison of the 1978 outburst and BO6 of Aql X-1. The 1978 outburst was shifted along the time axis to match the decay branch of BO6. The dashed and solid curves represent the smooth profiles. See Sect.3.3.1 for details.

\section{Discussion}

The method of the $\mathrm{O}-\mathrm{C}$ residuals enabled us to determine $T_{\mathrm{C}}$ and its large variations in Aql X-1 in spite of several missing outbursts which fall into intervals of poor coverage of the light curve. The character of the $\mathrm{O}-\mathrm{C}$ curves in Aql X-1 (Fig. 6) bears a large similarity to the dwarf novae (Vogt 1980; Šimon 2000a,b,c; Šimon 2001b), i.e. variations of $T_{\mathrm{C}}$ are large but generally not chaotic. Figure 6 displays beyond any doubt that the long-term trends can be resolved in the $\mathrm{O}-\mathrm{C}$ curves. The $\mathrm{O}-\mathrm{C}$ diagrams show that although $T_{\mathrm{C}}=211$ days is prevailing the evolution of $T_{\mathrm{C}}$ shows several large jumps. The mean $T_{\mathrm{C}}$ varied by more than $1: 2$ and the switches sometimes occurred within just a single epoch. No sign of $T_{\mathrm{C}}$ as short as 125 days, reported by Priedhorsky \& Terrell (1984) and Kitamoto et al. (1993), has been found.

Knowledge of the disk structure is important for the search for the underlying mechanism of $T_{\mathrm{C}}$ variations. Previous authors interpreted the outburst X-ray spectra of Aql X-1 as originating from several components: thermal components from the NS and from the accretion disk and a power-law component (Comptonized radiation from a corona) or a thermal bremsstrahlung component from hot plasma (Zhang et al. 1998; Cui et al. 1998). The thermal component is softer than the coronal component and should dominate in the $\mathrm{A}$ and $\mathrm{B}$ channels of $A S M / R X T E$. On the other hand, the non-thermal component is expected to contribute mainly to the emission detected in C channel. If $I_{\mathrm{A}}$ and $I_{\mathrm{B}}$ are taken as a measure of the mass accretion rate onto the NS, $\dot{m}_{\mathrm{NS}}$, then we can see from Figs. $1 \mathrm{~b}$ and 6 that very similar $T_{\mathrm{C}}$ 's can occur even if the X-ray intensities of the respective events are largely divergent, up to a factor of ten. This can suggest that the amount of matter, accreted onto the NS, largely differs from outburst to outburst. The FOs occupy a similar position in color-color diagram as the bright outbursts, which indicates that their low X-ray intensity is caused by a lower $\dot{m}_{\mathrm{NS}}$ rather than by a higher absorption (see also below). The decay branches of bright outbursts in Aql X-1 are mostly linear rather than exponential (Fig. 8). Following the model by King \& Ritter (1998), this implies that just the inner disk region is irradiated during the outburst. The disk is not depleted completely in this case because there is relatively much matter left in its outer region. Several closely-spaced outbursts can then occur and deplete the disk. Later, they can be replaced by a long quiescent interval during which the disk is refilled. One can then infer that $T_{\mathrm{C}}$ of Aql X-1 is governed at a large extent by the redistribution of matter in the disk rather than by the amount of mass accreted from the disk onto the NS during outburst. The irradiation influences just the inner region while the "clock" governing the length of $T_{\mathrm{C}}$ resides in the outer, non-irradiated disk parts. Aql X-1 displayed an unusual behaviour when it did not return to quiescence immediately after the decline from the outburst BO5. Two rebrightenings, between which $I_{\text {sum }}$ remained above the quiescent level, followed the main peak (Fig. 1a). The quiescent interval to the next outburst was considerably longer than the previous one. It can be inferred that this rebrightening affected the following outburst. The X-ray signal suggests that mass accretion onto the NS still continued. This could deplete more matter from the disk and, what is more important, change the mass distribution in it. 

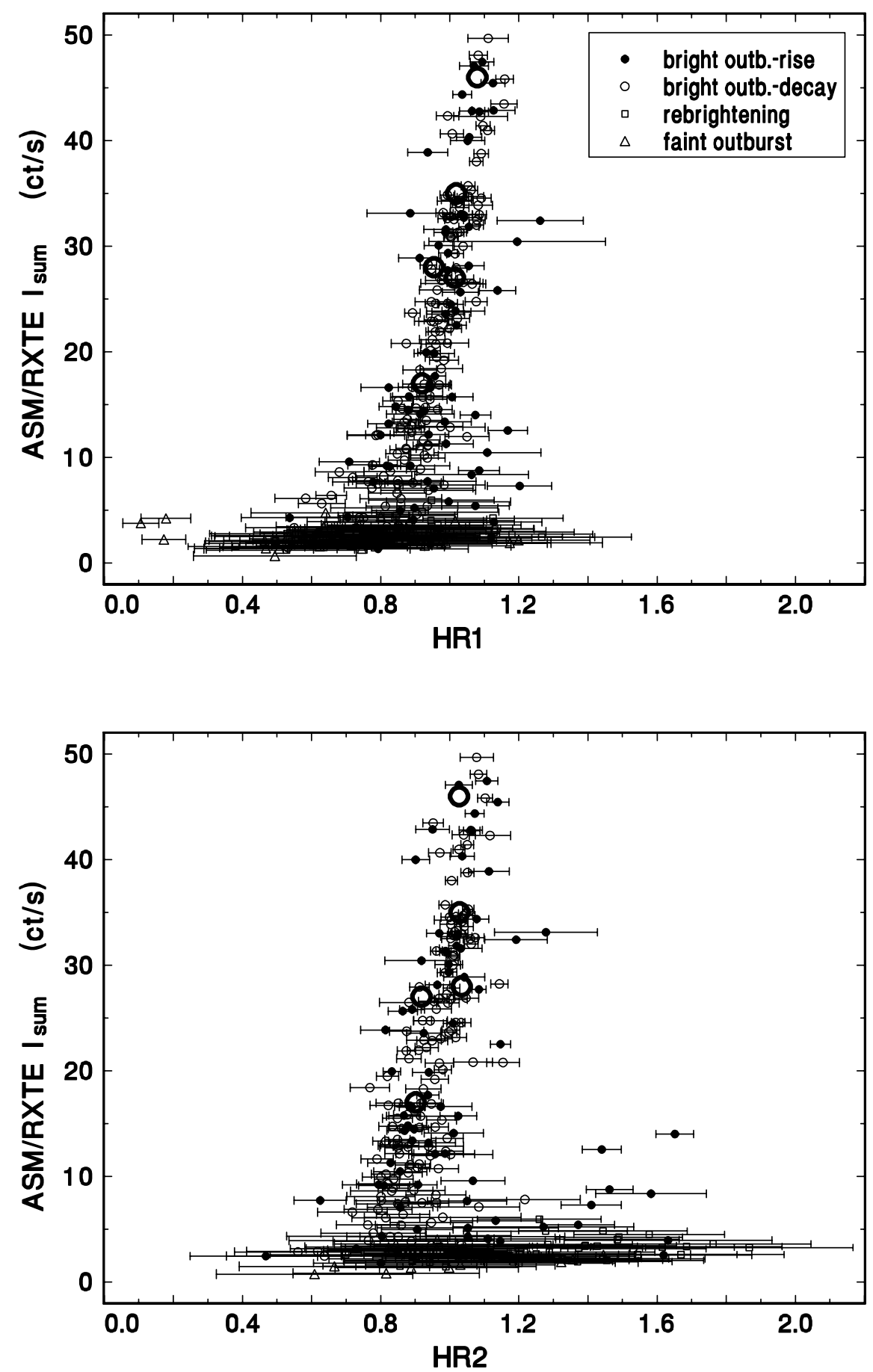

Fig. 11. The dependence of $H R 1$ and $H R 2$ on $I_{\text {sum }}$ in Aql X-1. The rising and decay branches of the bright outbursts, the FOs and the rebrightening of BO5 are resolved by the different symbols. The scales of the axes are identical for both diagrams. The large empty circles represent the maxima of the individual bright outbursts. See Sect. 3.4 for details.

The absolute brightness of Aql X-1 in the $V$-band during an outburst is $M_{V}(\max )=3.3$, from typical $m_{\max }=16.9$ (Fig. 1a). When this value is compared to the dwarf novae at the upper limit of $P_{\text {orb }}$ of CVs (GK Per $\left(M_{V}(\max )=1.1\right) ; \mathrm{DX}$ And $\left.\left(M_{V}(\max )=1.9\right)\right)$ it shows that Aql X-1 lies well within the values of $M_{V}(\max )$ which can be reached purely by the thermal instability, without strong irradiation by the NS. Again, this supports the conclusion that just the inner disk region is irradiated during outburst of Aql X-1 and viscous heating contributes significantly. BO3 which belongs to the segment with $T_{\mathrm{C}}=211$ days ( $E=44$ in Fig. 6 d) was classified as outside-in by Shahbaz et al. (1998a). This means that it was triggered in the outer disk region, presumably least influenced by the irradiation during the previous outburst. This event brings further evidence that at least during the rise the intrinsic luminosity of the disk (not caused by the reprocessing of X-rays) represented a 


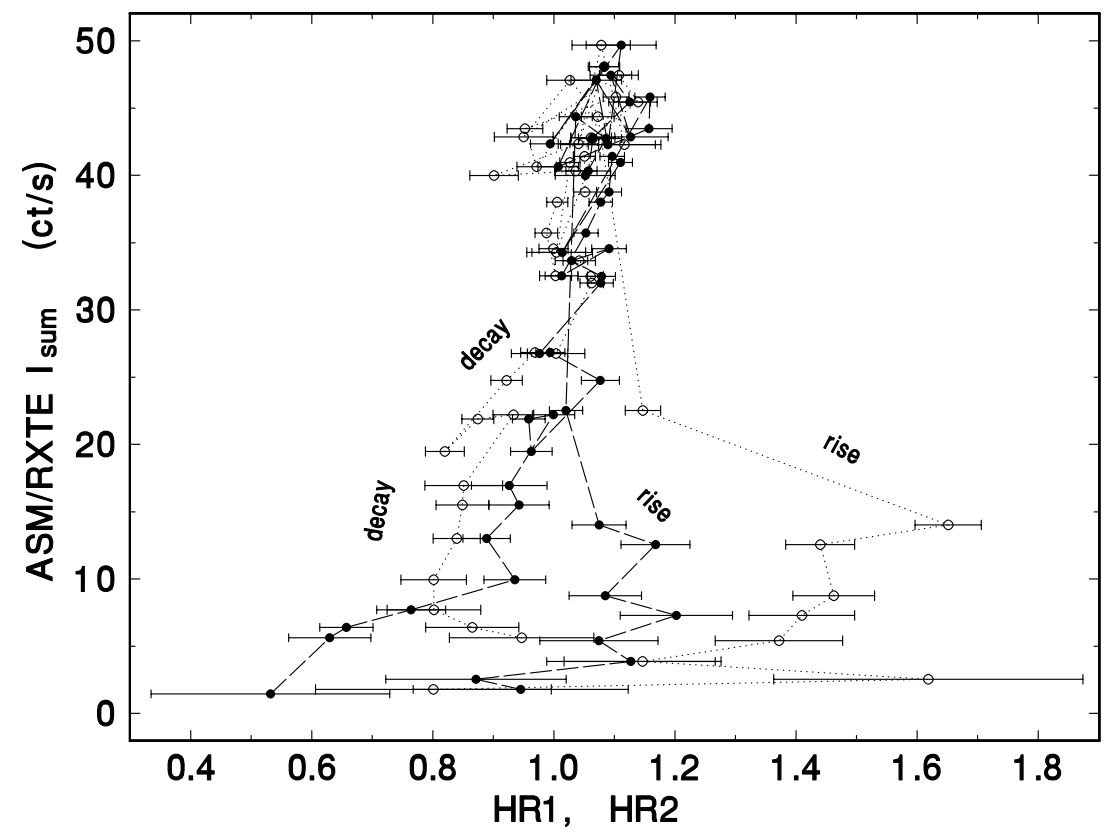

Fig. 12. The dependence of $H R 1$ (solid circles) and $H R 2$ (empty circles) on $I_{\text {sum }}$ of the outburst BO6. The rising and decay branches are labeled and the symbols are connected by the lines to trace the evolution of the whole event. See Sect. 3.4 for details.

large part of the system brightness. Also the lengths of $T_{\mathrm{C}}$, observed in Aql X-1, are comparable to those in CVs with long $P_{\text {orb }}\left(\mathrm{CH}\right.$ UMa: $T_{\mathrm{C}}=307-370$ days, Šimon 2000a; DX And: $T_{\mathrm{C}}=207-328$ days, Šimon 2000b; GK Per: $T_{\mathrm{C}}=400-1000$ days, e.g. Hudec 1981).

The study of the morphology of the $A S M / R X T E$ light curves revealed that although the respective bright outbursts in Aql X-1 differ in both their duration and $I_{\max }$, their rising and decay branches display remarkable similarities.

The shape of the rising branch remains very similar for the individual events and can be approximated by the exponential function. This suggests that the rate of increase of $\dot{m}_{\mathrm{NS}}$ is very similar for the respective events, regardless of the $I_{\max }$ of a given outburst. The thermal instability therefore appears to set in at very similar radii for all these events, as can be inferred from the models by Dubus et al. (2001). The $e$-folding time of 3.2 days would sort Aql X-1 among the faster rises in the histogram of X-ray novae by Chen et al. (1997). Only BO2 had an X-ray precursor (Fig. 9). This feature can arise if a sufficient amount of matter arrives in the inward propagating part of the heating front (Dubus et al. 2001).

The slope of the decay was found to be broken in most events, with the final phase having a steeper slope. The initial phase of the decays of some outbursts can be characterized even as a plateau, with just a slow decline. The profile of the final decay appears to be largely independent of the duration of the outburst and is linear for $I_{\text {sum }}>8-10 \mathrm{ct} / \mathrm{s}$ (Fig. 8a) while the exponential curve is a better approximation below this level (Fig. 8c). Such a profile is consistent with the detailed analytical model of the decay of a disk, whose inner region is irradiated by the central source while the outer parts are not (King 1998). This model predicts steep power-law decays which can be plausibly approximated by a linear function, except for the lowermost part of the X-ray light curve. The slope of the final decay is quite similar for the outbursts with largely different $I_{\max }$. This slope depends on the viscosity $\nu$ near the edge of the irradiated radius (King \& Ritter 1998), hence $\nu$ achieves very similar values for the respective events in Aql X-1. Since the linear decay can start from the different $I_{\text {sum }}$ 's it means that the disk mass at the onset of the linear phase can vary from outburst to outburst. Comparison with the 1978 outburst revealed that its smoothed decay branch bears a resemblance to the events, observed by $R X T E$, in both its shape and decay rate, hence $\nu$ is likely about the same in all these events. Our analysis offers an alternative interpretation of the shape of the decay branch of the bright outbursts in Aql X-1. Shahbaz et al. (1998b) suggested that the slope of the linear decay is smaller (i.e. the decay is slower) for the fainter events, suggesting thus their smaller $\nu$. We have shown that the decay branch of some outbursts consists of two parts, the first being slower, sometimes even displaying a plateau, and the later phase being more rapid. For the fainter events the rapid part of the decay occurs at relatively weak signals and may not be easily resolvable. Nevertheless, the procedure of matching the template (Fig. 8) has shown that the lower parts of both events, analyzed by Shahbaz et al. (1998b), match the template quite well and that their decay curves can be classified as broken. Shahbaz et al. (1998b) analyzed two events with relatively low $I_{\max }$. The profile of BO3 

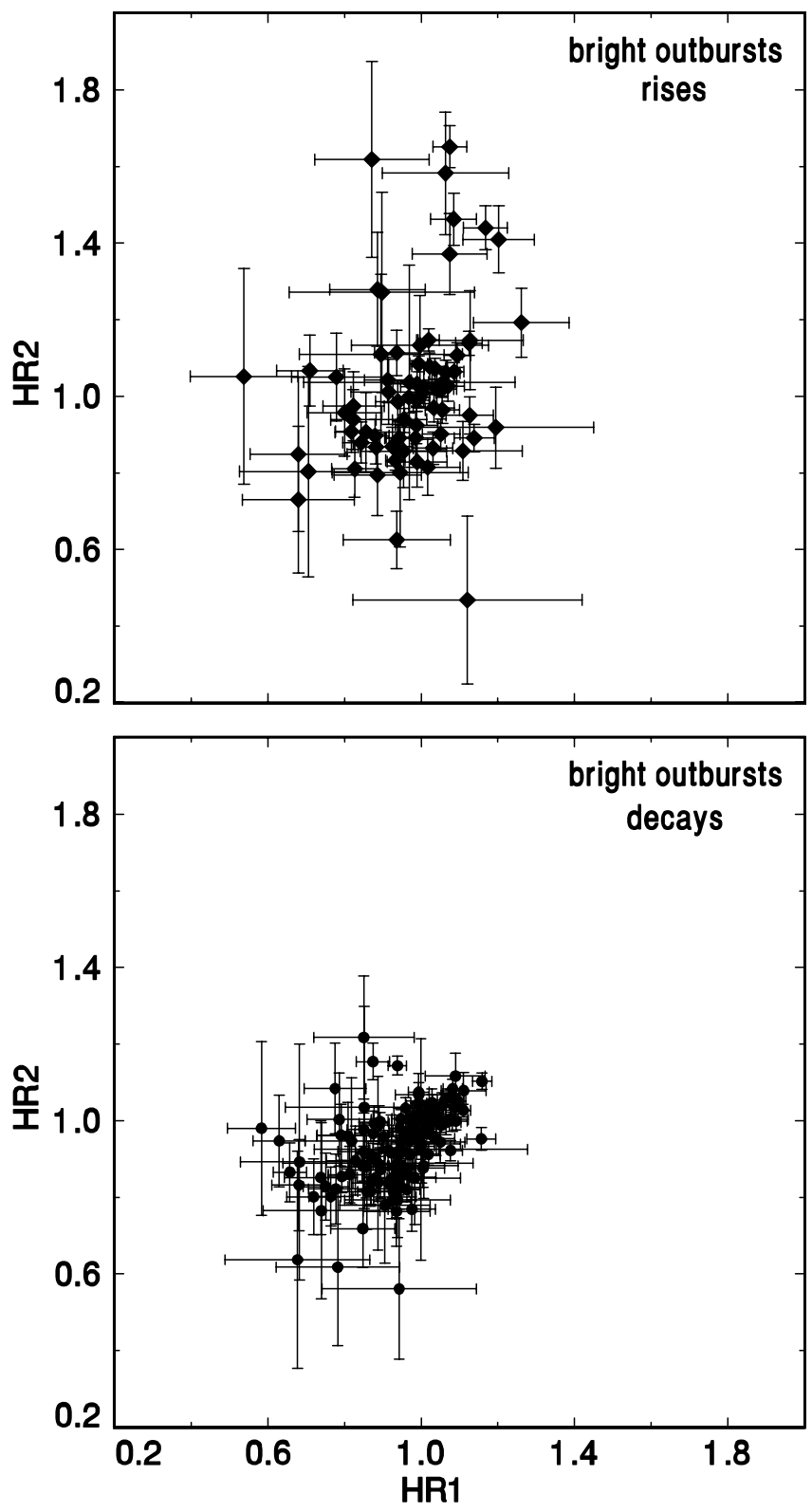
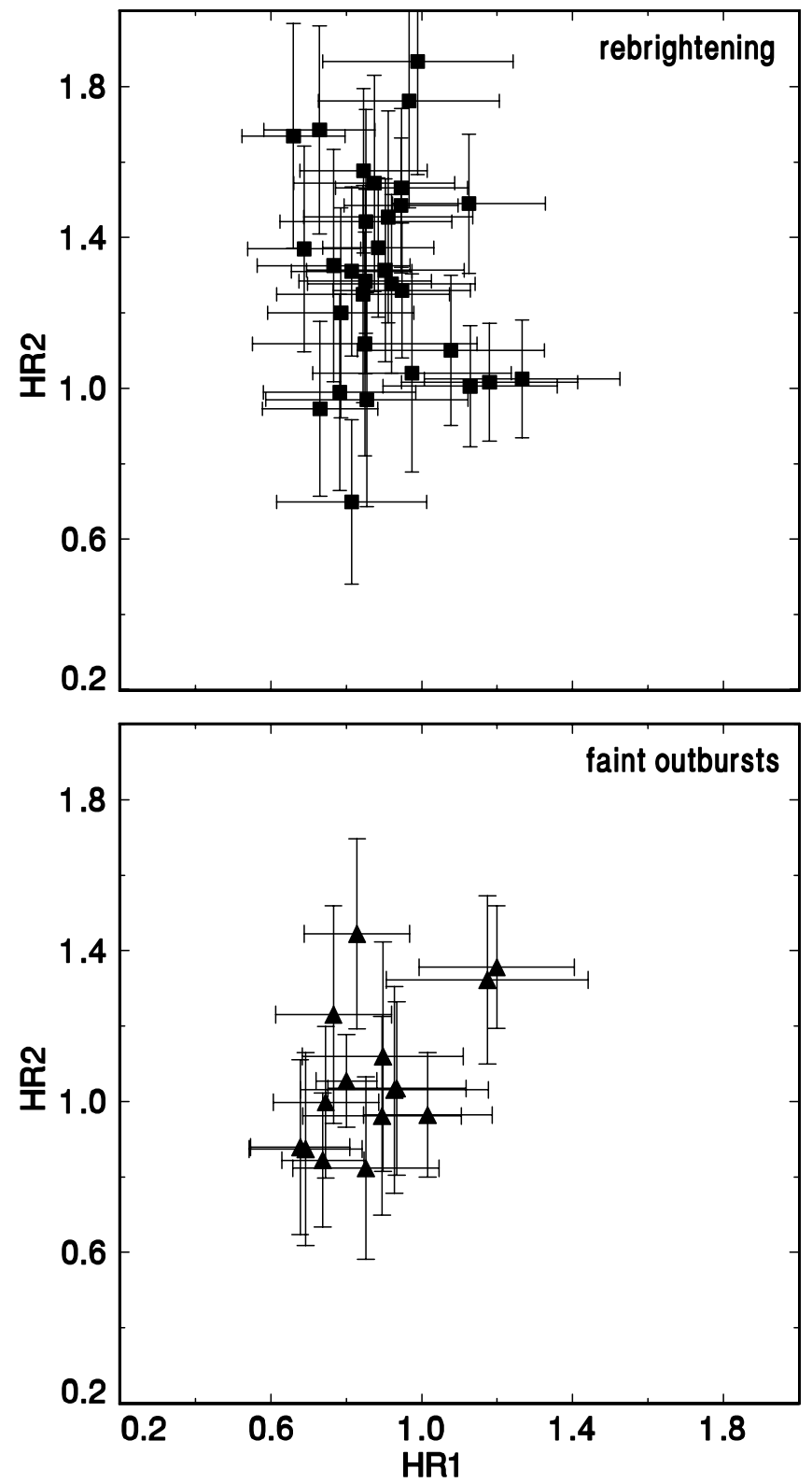

Fig. 13. X-ray color-color diagrams for Aql X-1. The panel labeled "rebrightening" shows the colors of the event which followed the decay from the main peak of the outburst BO5. See Sect. 3.4 for details.

which they used displays a change of the slope of both the rising and decay branches at $I_{\text {sun }} \approx 10 \mathrm{ct} / \mathrm{s}$, the upper part of the curve being much flatter (Fig. 2). It is therefore quite reasonable to characterize its upper part as a plateau, more rounded than in $\mathrm{BO} 4$.

In principle, there is one more process which can influence the late decay. When the mass accretion rate, $\dot{m}$, decreases below a certain level then the magnetic field of the NS will prevent further accretion onto the NS via the propeller effect. Zhang et al. (1998) attributed the onset of this phase to the hardening of the PCA count rate which occurred for $I_{\text {sum }} \approx 3-4 \mathrm{ct} / \mathrm{s}$. This means that the propeller effect does not significantly affect the decay profiles, analyzed in Fig. 8. However, it is interesting to note that the typical $I_{\text {sum }}$ of the FOs and the rebrightening of BO5 (about $3-5 \mathrm{ct} / \mathrm{s}$ ) is just above the inferred limit for the propeller effect.

The bright outbursts of Aql X-1 which reached lower $I_{\max }$ tend to have softer $H R 1$ and to some extent also $H R 2$ at maximum (Fig. 11). Usually, both $H R 1$ and $H R 2$ settle at some level of the general track for the bright outbursts in Fig. 11 when the intensity is near $I_{\max }$. This can be explained if the high $\dot{m}$, giving rise to the brighter $\mathrm{X}$-ray events, leads to a higher disk (or disk+NS) temperature. We have found a remarkable asymmetry (hysteresis) between the evolution of both $H R 1$ and HR2 during the rise and decay, particularly prominent during the outburst BO6. The transient increase of HR2 during the rise 
to some outbursts can be interpreted in terms of a more rapid increase of the coronal emission in comparison with the thermal disk+NS component.

X-ray color-color diagrams in Fig. 13 show that the four groups differ from each other by their HR2's more than by HR1's. The decay branches of the bright outbursts form a closed, elongated group with a small scatter. The shape of this group resembles an atoll track. It can be seen from a comparison with Fig. 11 that the transition along this track is gradual, and can be attributed to a "banana" branch. Previous evidence for the atoll classification was presented by e.g. Reig et al. (2000). The decays have the softest mean HR2 of the four panels in Fig. 13. All these findings suggest that both the temperature of the inner disk region and the NS achieve quite similar values and evolve in a quite similar manner during the decline of the individual outbursts. At the same time, the contribution of the non-thermal component has already decreased. The rising branches of the bright outbursts have about the same mean $H R 1$ and $H R 2$ as the decays but they display a larger scatter along the $H R 2$ axis, caused by the hysteresis.

The FOs appear to have their HR1 and HR2 comparable to those of the bright outbursts or they may be just slightly harder. This speaks in favour of an intrinsically lower X-ray intensity during the FOs rather than their higher absorption in comparison with the bright outbursts. Also since the orbital inclination of Aql X-1 is relatively low $\left(i \approx 36^{\circ}-55^{\circ}\right.$; Welsh et al. 2000) the obscuration of the NS by the disk is expected to be rather small. The rebrightening of the outburst BO5 is considerably harder than the bright outbursts, when crossing a comparable $I_{\text {sum }}$, in general. Therefore, the track of this rebrightening in the color-color diagram is not a continuation of the decay from the main peak. One can infer that the non-thermal component played a big role during this rebrightening. However, it is rather surprising that the position of the FOs is different from the rebrightening of BO5 although their $I_{\text {sum }}$ 's are comparable. A tentative explanation can assume the plasma which remained in the inner disk region after the main peak of BO5. This plasma becomes very hot in the propeller regime (Wang \& Robertson 1985; Zhang et al. 1998). The main peak of BO5 has the shortest duration of the five well-covered bright outbursts. The rebrightening therefore might include continuing injections of plasma into the inner disk and this region could balance between the accretion onto the NS and the propeller regime.

Pronounced fluctuations of the X-ray intensity were apparent around $I_{\max }$ of the most intense outburst BO6. Examination of the light curves of the individual bright outbursts revealed that the events with smaller $I_{\max }$ generally had smoother profiles. This may suggest that the underlying instability can develop only when $\dot{m}_{\mathrm{NS}}$ exceeds some minimum level. Similar fluctuations with an even larger amplitude were also reported for the 1978 outburst which reached the flux of about 1 Crab (Charles et al. 1980). Since the fluctuations of BO6were the most prominent in the $\mathrm{C}$ channel, the contribution from the power-law component may be substantial. A similar behaviour, that is, a higher variability of the harder X-ray component than the soft component, appears to be common in the SXTs (Chen et al. 1997).

\section{Conclusions}

We have presented a comprehensive analysis of the longterm activity of the NS SXT Aql X-1. We have resolved that variations of $T_{\mathrm{C}}$ are large, but generally not chaotic and resolved long-term trends can be. The prevailing value of $T_{\mathrm{C}}$ is 211 days, in variance with that determined before. The extensive analysis of the $A S M / R X T E$ data for several outbursts of Aql X-1 enabled us to resolve features in their X-ray light curves with an accuracy never achieved before. Although the respective outbursts differ in both their duration and $I_{\max }$, the profiles of their branches and the evolution of $H R 1$ and $H R 2$ display remarkable similarities. We argue that just the inner disk is irradiated during outburst and that viscous heating plays an important role.

Acknowledgements. This research has made use of NASA's Astrophysics Data System Abstract Service and the observations provided by the $A S M / R X T E$ team. I thank Dr. Hudec for reading the manuscript and for his comments. I am indebted to Dr. Harmanec for providing me with the program HEC 13. The support by the post-doctoral grant 205/00/P013 of the Grant Agency of the Czech Republic and the project ESA PRODEX INTEGRAL 14527 is acknowledged.

\section{References}

Charles, P. A., Thorstensen, J. R., Bowyer, S., et al. 1980, ApJ, 237, 15480

Chevalier, C., \& Ilovaisky, S. A. 1991, A\&A, 251, L11

Chevalier, C., Ilovaisky, S. A., Leisy, P., \& Patat, F. 1999, A\&A, 347, L51

Chen, W., Shrader, C. R., \& Livio, M. 1997, ApJ, 491, 312

Cui, W., Barret, D., Zhang, S. N., et al. 1998, ApJ, 502, L49

Dubus, G., Hameury, J.-M., \& Lasota, J.-P. 2001, A\&A, 373, 251

Harmon, B. A., Wilson, C. A., Tavani, M., et al. 1996, A\&AS, 120,197

Hudec, R. 1981, Bull. Astron. Inst. Czechosl., 32, 93

King, A. R. 1998, MNRAS, 296, L45

King, A. R., \& Ritter, H. 1998, MNRAS, 293, L42

Kitamoto, S., Tsunemi, H., \& Miyamoto, S. 1993, ApJ, 403, 315

Koyama, K., Inoue, H., Makishima, K., et al. 1981, ApJ, 247, L27

Priedhorsky, W. C., \& Terrell, J. 1984, ApJ, 280, 661

Reig, P., Méndez, M., van der Klis, M., \& Ford, E. C. 2000, ApJ, 530, 916 
Shahbaz, T., Bandyopadhyay, R. M., Charles, P. A., et al. Šimon, V., 2001b, Proc. of the Conference, The Physics 1998a, MNRAS, 300, 1035

Shahbaz, T., Charles, P. A., \& King, A. R. 1998b, MNRAS, 301,382

Šimon, V. 2000a, A\&A, 354, 103

Šimon, V. 2000b, A\&A, 360, 627

Šimon, V. 2000c, A\&A, 364, 694

Šimon, V. 2001a, Proc. of the Conference, The Physics of Cataclysmic Variables and Related Objects, ASP Conf. Ser., in press

of Cataclysmic Variables and Related Objects, ASP Conf. Ser., in press

van Paradijs, J. 1996, ApJ, 464, L139

Vogt, N. 1980, A\&A, 88, 66

Vondrák, J. 1969, Bull. Astron. Inst. Czechosl., 20, 349

Vondrák, J. 1977, Bull. Astron. Inst. Czechosl., 28, 84

Wang, Y.-M., \& Robertson, J. A. 1985, A\&A, 151, 361

Welsh, W. F., Robinson, E. L., \& Young, P. 2000, AJ, 120, 943

Zhang, S. N., Yu W., \& Zhang, W. 1998, ApJ, 494, L71 